\title{
K-THEORY OF PSEUDODIFFERENTIAL OPERATORS WITH SEMI-PERIODIC SYMBOLS
}

\author{
SEVERINO T. MELO AND CÍNTIA C. SILVA
}

\begin{abstract}
Let $\mathfrak{A}$ denote the $\mathrm{C}^{*}$-algebra of bounded operators on $L^{2}(\mathbb{R})$ generated by: (i) all multiplications $a(M)$ by functions $a \in C[-\infty,+\infty]$, (ii) all multiplications by $2 \pi$-periodic continuous functions, and (iii) all operators of the form $F^{-1} b(M) F$, where $F$ denotes the Fourier transform and $b \in C[-\infty,+\infty]$. A given $A \in \mathfrak{A}$ is a Fredholm operator if and only if $\sigma(A)$ and $\gamma(A)$ are invertible, where $\sigma$ denotes the continuous extension of the usual principal symbol, while $\gamma$ denotes an operator-valued "boundary principal symbol" (the "boundary" here consists of two copies of the circle, one at each end of the real line). We give two proofs of the fact that $K_{0}(\mathfrak{A})$ is isomorphic to $\mathbb{Z}$ and that $K_{1}(\mathfrak{A})$ is isomorphic to $\mathbb{Z} \oplus \mathbb{Z}$. We do it first by computing the connecting mappings in the six-term exact sequence associated to $\sigma$. For the second proof, we show that the image of $\gamma$ is isomorphic to the direct sum of two copies of the crossed product $C[-\infty,+\infty] \times{ }_{\alpha} \mathbb{Z}$, where $\alpha$ denotes the translation-by-one automorphism. Its K-theory can be computed using the Pimsner-Voiculescu exact sequence, and that information suffices for the analysis of the standard cyclic exact sequence associated to $\gamma$.
\end{abstract}

2000 Mathematics Subject Classification: 46L80, 47G30 (19K56, 47A53, 47L80).

\section{INTRODUCTION}

Let $\mathfrak{A}$ denote the smallest $\mathrm{C}^{*}$-subalgebra of the algebra of all bounded operators on $L^{2}(\mathbb{R})$ containing:

(1) every multiplication $a(M)$ by an $a \in C[-\infty,+\infty]$, where $[-\infty,+\infty]$ denotes the two-point compactification of $\mathbb{R}$ (i.e., $a$ is continuous on $\mathbb{R}$ and has limits at $+\infty$ and at $-\infty)$,

(2) every multiplication by a $2 \pi$-periodic continuous function, and

(3) every Fourier multiplier of the form $b(D)=F^{-1} b(M) F$, where $F$ denotes the Fourier transform and $b \in C[-\infty,+\infty]$.

The structure of $\mathfrak{A}$ is described in the following four theorems, which are rephrased versions of results first appearing in [7, 13, where this algebra is regarded as a particular comparison algebra; i.e., as a member of a certain class, defined by Cordes [4, of $\mathrm{C}^{*}$-algebras generated by pseudodifferential operators. These results are partially reproven in the first chapter of [26, avoiding reference to general results on comparison algebras.

Theorem 1. Let $X$ denote the subset of $[-\infty,+\infty] \times \mathbb{S}^{1}$ of all points $\left(x, e^{i \theta}\right)$ such that $|x|=\infty$ or $x=\theta \in \mathbb{R}$. There exists a surjective $C^{*}$-algebra homomorphism

$$
\begin{aligned}
\sigma: \mathfrak{A} & \longrightarrow C(X \times\{-\infty,+\infty\}) \\
A & \longmapsto \sigma_{A}
\end{aligned}
$$

such that, for each $m=\left(x, e^{i \theta}\right) \in X$, we have: 
(i) $\sigma_{a(M)}(m,+\infty)=\sigma_{a(M)}(m,-\infty)=a(x)$ if $a \in C[-\infty,+\infty]$,

(ii) $\sigma_{a(M)}(m,+\infty)=\sigma_{a(M)}(m,-\infty)=a(\theta)$ if a is a continuous $2 \pi$-periodic function on $\mathbb{R}$, and

(iii) $\sigma_{b(D)}(m,+\infty)=b(+\infty)$ and $\sigma_{b(D)}(m,-\infty)=b(-\infty)$ if $b \in C[-\infty,+\infty]$.

Corollary 1. Let $\mathfrak{E}$ denote the kernel of $\sigma$. The mapping

$$
\begin{aligned}
& \mathfrak{A} / \mathfrak{E} \longrightarrow C(X \times\{-\infty,+\infty\}) \\
& {[A]_{\mathfrak{E}} \longmapsto \sigma_{A}}
\end{aligned}
$$

is a $C^{*}$-algebra isomorphism.

For each $\varphi \in \mathbb{R}$, let $U_{\varphi}$ denote the operator on $L^{2}\left(\mathbb{S}^{1}\right)$ given by $U_{\varphi} f(z)=$ $z^{-\varphi} f(z), z \in \mathbb{S}^{1}$, and let $Y_{\varphi}$ denote the operator on $\ell^{2}(\mathbb{Z})$ obtained by conjugating $U_{\varphi}$ with the discrete Fourier transform. Then, $Y_{\varphi}$ is a smooth family of unitary operators such that, for all $j \in \mathbb{Z}, Y_{j}\left(\left(u_{k}\right)_{k \in \mathbb{Z}}\right)=\left(u_{j+k}\right)_{k \in \mathbb{Z}}$.

Below we denote by $S^{*} \mathbb{S}^{1}=\mathbb{S}^{1} \times\{-1,+1\}$ the co-sphere bundle over the unit circle $\mathbb{S}^{1}$, by $\mathfrak{B}$ the algebra of all bounded operators on $\ell^{2}(\mathbb{Z})$, and the identity operator on $\ell^{2}(\mathbb{Z})$ or on $L^{2}(\mathbb{R})$ by $I$.

Theorem 2. There exists a $C^{*}$-algebra homomorphism

$$
\begin{aligned}
\gamma: \mathfrak{A} & \longrightarrow C\left(S^{*} \mathbb{S}^{1}, \mathfrak{B}\right) \\
A & \longmapsto \gamma_{A}
\end{aligned}
$$

such that, for each $v=\left(e^{2 \pi i \varphi}, \pm 1\right) \in S^{*} \mathbb{S}^{1}$, we have:

(i) $\gamma_{a(M)}(v)=a( \pm \infty) I$ if $a \in C[-\infty,+\infty]$,

(ii) $\gamma_{e_{k}(M)}(v)=Y_{-k}$, for $e_{k}(x)=e^{i k x}, k \in \mathbb{Z}$, and

(iii) $\gamma_{b(D)}(v)=Y_{\varphi} M_{\varphi}^{b} Y_{-\varphi}$, if $b \in C[-\infty,+\infty]$, with $M_{\varphi}^{b}$ defined by $M_{\varphi}^{b}\left(\left(a_{j}\right)_{j \in \mathbb{Z}}\right)=$ $\left(b(j-\varphi) a_{j}\right)_{j \in \mathbb{Z}}$.

It is easy to show, and this is part of the statement of Theorem 2 that the function $\mathbb{R} \ni \varphi \mapsto Y_{\varphi} M_{\varphi}^{b} Y_{-\varphi} \in \mathfrak{B}$ is 1-periodic if $b \in C[-\infty,+\infty]$.

Let $\mathcal{A}^{\sharp}$ denote the algebra of bounded functions on $\mathbb{R}$ generated by $C[-\infty,+\infty]$ and by all $2 \pi$-periodic continuous functions. It is closed. Let $C_{0}(\mathbb{R})$ denote the algebra of all continuous functions with zero limits at $+\infty$ and $-\infty$.

Let $\mathfrak{K}_{\mathbb{R}}$ denote the ideal of compact operators on $L^{2}(\mathbb{R})$ and $\mathfrak{K}_{\mathbb{Z}}$ denote the ideal of compact operators on $\ell^{2}(\mathbb{Z})$.

Theorem 3. A dense *-subalgebra of the kernel of $\sigma$ is given by the linear span of all operators of the form $a(M) b(D)+K$, with $a \in \mathcal{A}^{\sharp}, b \in C_{0}(\mathbb{R})$, and $K \in \mathfrak{K}_{\mathbb{R}}$. The image of the restriction of $\gamma$ to $\mathfrak{E}$ is equal to $C\left(S^{*} \mathbb{S}^{1}, \mathfrak{K}_{\mathbb{Z}}\right)$ and the mapping

$$
\begin{aligned}
& \mathfrak{E} / \mathfrak{K}_{\mathbb{R}} \longrightarrow C\left(S^{*} \mathbb{S}^{1}, \mathfrak{K}_{\mathbb{Z}}\right) \\
& {[A]_{\mathfrak{K}_{\mathbb{R}}} \longmapsto \gamma_{A}}
\end{aligned}
$$

is a $C^{*}$-algebra isomorphism.

Theorem 4. The intersection of the kernels of $\sigma$ and $\gamma$ is equal to $\mathfrak{K}_{\mathbb{R}}$. Moreover, a given $A \in \mathfrak{A}$ is a Fredholm operator if and only if $\sigma(A)$ and $\gamma(A)$ are invertible.

A similar Fredholm criterion had been given earlier by Rabinovich 24 for an algebra larger than this. The above stated results were later extended 14 to a comparison algebra on a cylinder of the form $\mathbb{R} \times B$, where $B$ is a compact Riemannian manifold. 
In this paper, we give two proofs that $K_{0}(\mathfrak{A}) \cong \mathbb{Z}$ and $K_{1}(\mathfrak{A}) \cong \mathbb{Z} \oplus \mathbb{Z}$ (Theorem [6): by computing the connecting mappings in the standard K-theory cyclic exact sequences associated to the two $\mathrm{C}^{*}$-algebra short exact sequences induced by the principal symbol $\sigma$ and by the boundary principal symbol $\gamma$. The crucial step in the second computation is finding a useful characterization of the image of $\gamma$, $\operatorname{Im} \gamma$, and computing its K-groups. We do that by showing that $\operatorname{Im} \gamma$ is isomorphic to the direct sum of two copies of the crossed product $C[-\infty,+\infty] \times_{\alpha} \mathbb{Z}$, where $\alpha$ denotes the translation-by-one automorphism. Its K-theory can then be computed using the Pimsner-Voiculescu exact sequence.

Many other $\mathrm{C}^{*}$-algebras generated by zero-order pseudodifferential operators (see 3, 15, 11, 12, 14, 15, 20, for example, but this is a very incomplete list) have basically the same structure as our $\mathfrak{A}$; i.e., a two-step composition series of the form

$$
\mathfrak{K} \subset \mathfrak{E} \subset \mathfrak{A},
$$

with $\mathfrak{A} / \mathfrak{E}$ commutative and

$$
\mathfrak{E} / \mathfrak{K} \cong C_{0}(Y) \otimes \mathfrak{K},
$$

for some locally compact Hausdorff space $Y$. In (3), $\mathfrak{K}$ is the ideal of compact operators on the Hilbert space where $\mathfrak{A}$ acts, while in (4) $\mathfrak{K}$ should rather be regarded as the compact ideal on a Hilbert space $H_{b}$ somehow associated to a "boundary", which can be the actual boundary of a manifold with boundary in some cases. In most examples, the Fredholm property for an arbitrary operator $A \in \mathfrak{A}$ is equivalent to the invertibility of $\sigma(A)$ and $\gamma(A)$; where $\sigma$ (the principal symbol) is the composition of the Gelfand mapping for $\mathfrak{A} / \mathfrak{E}$ with the canonical projection on the quotient, and $\gamma$ (the boundary principal symbol) is a $\mathrm{C}^{*}$-algebra homomorphism

$$
\gamma: \mathfrak{A} \longrightarrow C_{0}(Y) \otimes \mathfrak{B}
$$

which extends the composition of the isomorphism in (4) with the quotient projection. Here $\mathfrak{B}$ denotes the algebra of bounded operators on $H_{b}$.

Given an algebra $\mathfrak{A}$ with such a structure, one might ask what pieces of information are needed to compute its K-theory, how to explicitly describe generators for its K-groups. A detailed understanding of the example treated in this paper will hopefully offer some insight for this more abstract question.

A strategy parallel to that of Section 2 was used by Melo, Nest and Schrohe in [15]. In that paper, finding convenient descriptions of the image and the kernel of the boundary principal symbol was the key for a successful analysis of the cyclic exact sequence associated to $\gamma$, which lead to the computation of the K-theory of Boutet de Monvel's algebra on a compact manifold whose boundary is nonempty and has torsion-free K-groups. With the help of K-theory tools a little more sofisticated than the standard cyclic sequence, but still relying on the same description of the image and kernel of $\gamma$, that torsion-free assumption was later removed by Melo, Schick and Schrohe [16]. For the computation of the K-theory of the comparison algebra with periodic multiplications on a cylinder studied in 14], also the approach of finding a good description of the image of the boundary principal symbol seems to be more promissing than trying to compute directly the connecting mappings in the cyclic exact sequence associated to the principal symbol. 
Using the language of groupoids, Monthubert and Nistor [18] extended Atiyah and Singer's 1] definition of topological index for manifolds with corners and used it to compute the connecting mappings in the K-theory cyclic exact sequence associated to the principal symbol of Melrose's b-calculus. For the algebra of suspended pseudo-differential operators, also introduced by Melrose [17, Moroianu [20] found an interesting connection between the Fredholm index of Dirac operators and the exponential mapping (the index mapping vanishes in this case) in the cyclic sequence associated to the principal symbol.

We use twice in this paper the following index theorem due to Cordes, Herman and Power 6, 23.

Theorem 5. Let $\mathfrak{C}$ denote the $C^{*}$-algebra of bounded operators on $L^{2}(\mathbb{R})$ generated by all operators of type (1) or (3) (listed at the beginning of this paper). Let $\mathbb{M}$ denote the boundary points of the square compactification of $\mathbb{R}^{2}$,

$$
\mathbb{M}=\{(x, \xi) \in[-\infty,+\infty] \times[-\infty,+\infty] ;|x|+|\xi|=\infty\} .
$$

The assignment $a(M) b(D) \mapsto a(x) b(\xi)$ extends to a surjective $C^{*}$-algebra homomorphism $\bar{\sigma}: \mathfrak{C} \rightarrow C(\mathbb{M})$ with kernel $\mathfrak{K}_{\mathbb{R}}$. A given $A \in \mathfrak{C}$ is a Fredholm operator if and only if $\bar{\sigma}(A)$ does not vanish at any point of $\mathbb{M}$; in which case, its Fredholm index is equal to the winding number of $\bar{\sigma}(A)$ (if $\mathbb{M}$ is identified with the circle in the canonical way).

\section{THE PRINCIPAL-SYMBOL EXACT SEQUENCE}

Before analysing the K-theory cyclic exact sequence associated to

$$
0 \rightarrow \mathfrak{E} / \mathfrak{K}_{\mathbb{R}} \rightarrow \mathfrak{A} / \mathfrak{K}_{\mathbb{R}} \rightarrow \mathfrak{A} / \mathfrak{E} \rightarrow 0,
$$

we need first to describe the K-groups of $C(X)$, since it follows immediately from Corollary 1 that $\mathfrak{A} / \mathfrak{E} \cong C(X) \oplus C(X)$.

Choose a smooth non-increasing function $c: \mathbb{R} \rightarrow[0,1]$ such that $c(x)=1$ if $x \leq-1 / 5$ and $c(x)=0$ if $x \geq 1 / 5$, and let $b=1-c$. Later it will be clear why we require $c$ and $b$ to be constant outside this narrow interval. At first it would suffice to suppose only that $c(-\infty)=b(+\infty)=1$. Define also

$$
l(x)=\left\{\begin{array}{ll}
e^{i x} & \text { if } x \geq 0 \\
1 & \text { if } x<0
\end{array} \text { and } \tilde{l}(x)=\left\{\begin{array}{ll}
1 & \text { if } x \geq 0 \\
e^{i x} & \text { if } x<0
\end{array} .\right.\right.
$$

We may regard $\mathbb{R}$ as an open dense subset of $X$ (the precise statement is: the mapping $x \mapsto\left(x, e^{i x}\right)$ is a homeomorphism of $\mathbb{R}$ onto an open dense subset of $\left.X\right)$, and then denote also by $l$ and $\tilde{l}$ their unique continuous extensions to $X$.

Given a projection $p$ in a $\mathrm{C}^{*}$-algebra $A$, and a unitary $u$ in $A$ or in its unitization $A^{+}$, we denote by $[p]_{0}$ and $[u]_{1}$ the elements they represent, respectively, in $K_{0}(A)$ and in $K_{1}(A)$.

Given $f$ and $g$ in $C\left(\mathbb{S}^{1}\right)$, we denote by $(f, g)$ the function on $\{-\infty,+\infty\} \times \mathbb{S}^{1}$ which is $f$ over $-\infty$ and $g$ over $+\infty$, and denote by $z$ the identity function on $\mathbb{S}^{1}$. Then, for example, $(1, z)$ denotes the function which is constant and equal to 1 on $\{-\infty\} \times \mathbb{S}^{1}$ and maps $(+\infty, z)$ to $z$ for all $z \in \mathbb{S}^{1}$. Analogous notation will be used for operator-valued funcions on $S^{*} \mathbb{S}^{1}$ and also for functions on $X \times\{-\infty,+\infty\}$.

Proposition 1. $K_{0}(C(X))=\mathbb{Z}[1]_{0}$ and $K_{1}(C(X))=\mathbb{Z}[l]_{1} \oplus \mathbb{Z}[\tilde{l}]_{1}$. 
Proof: Let us consider the exact sequence

$$
0 \rightarrow C_{0}(\mathbb{R}) \rightarrow C(X) \rightarrow C\left(\{-\infty,+\infty\} \times \mathbb{S}^{1}\right) \rightarrow 0
$$

defined by the restriction mapping $C(X) \rightarrow C\left(\{-\infty,+\infty\} \times \mathbb{S}^{1}\right)$. We know that: $K_{0}\left(C_{0}(\mathbb{R})\right)=0, K_{1}\left(C_{0}(\mathbb{R})\right)=\mathbb{Z}\left[e^{2 \pi i b}\right]_{1}$,

$$
K_{0}\left(C\left(\{-\infty,+\infty\} \times \mathbb{S}^{1}\right)\right)=\mathbb{Z}[(1,0)]_{0} \oplus \mathbb{Z}[(0,1)]_{0},
$$

and

$$
K_{1}\left(C\left(\{-\infty,+\infty\} \times \mathbb{S}^{1}\right)\right)=\mathbb{Z}[(\mathbf{z}, 1)]_{1} \oplus \mathbb{Z}[(1, \mathbf{z})]_{1} .
$$

The standard $K$-theory cyclic sequence associated to (6) then becomes

$$
\begin{array}{ccccc}
0 & \longrightarrow & K_{0}(C(X)) & \longrightarrow & \mathbb{Z}[(1,0)]_{0} \oplus \mathbb{Z}[(0,1)]_{0} \\
\uparrow \delta_{1} & & & \downarrow \delta_{0} \\
\mathbb{Z}[(\mathbf{z}, 1)]_{1} \oplus \mathbb{Z}[(1, \mathbf{z})]_{1} & \longleftarrow & K_{1}(C(X)) & \longleftarrow & \mathbb{Z}\left[e^{2 \pi i b}\right]_{1} .
\end{array}
$$

The upper-right horizontal arrow in (7) maps $[1]_{0}$ to $[(1,1)]_{0}$, while the lower-left one maps $[l]_{1}$ to $[(1, \mathbf{z})]_{1}$ and $[\tilde{l}]_{1}$ to $[(\mathbf{z}, 1)]_{1}$. The restriction of $b$ to $\{-\infty,+\infty\} \times \mathbb{S}^{1}$ is $(0,1)$. It then follows from [25, 12.2.2] that $\delta_{0}\left([(0,1)]_{0}\right)=-\left[e^{2 \pi i b}\right]_{1}$. Analogously we get $\delta_{0}\left([(1,0)]_{0}\right)=-\left[e^{2 \pi i c}\right]_{1}$. Noticing that $\left[e^{2 \pi i b}\right]_{1}=-\left[e^{2 \pi i c}\right]_{1}$, the proof can now be finished by elementary algebraic arguments.

Defining $A_{1}=l(M) b(D)+c(D), A_{2}=\tilde{l}(M) b(D)+c(D), A_{3}=b(D)+l(M) c(D)$ and $A_{4}=b(D)+\tilde{l}(M) c(D)$, we have $\sigma_{A_{1}}=(1, l), \sigma_{A_{2}}=(1, \tilde{l}), \sigma_{A_{3}}=(l, 1)$ and $\sigma_{A_{4}}=(\tilde{l}, 1)$. Denoting by $[B]_{\mathfrak{E}}$ the class in $\mathfrak{A} / \mathfrak{E}$ of a $B \in \mathfrak{A}$, we obtain from the isomorphism (1) and from Proposition [1]

$$
K_{1}(\mathfrak{A} / \mathfrak{E})=\mathbb{Z}\left[\left[A_{1}\right]_{\mathfrak{E}}\right]_{1} \oplus \mathbb{Z}\left[\left[A_{2}\right]_{\mathfrak{E}}\right]_{1} \oplus \mathbb{Z}\left[\left[A_{3}\right]_{\mathfrak{E}}\right]_{1} \oplus \mathbb{Z}\left[\left[A_{4}\right]_{\mathfrak{E}}\right]_{1} .
$$

Since $\sigma_{b(D)}=(0,1)$ and $\sigma_{c(D)}=(1,0)$, we also get:

$$
K_{0}(\mathfrak{A} / \mathfrak{E})=\mathbb{Z}\left[[b(D)]_{\mathfrak{E}}\right]_{0} \oplus \mathbb{Z}\left[[c(D)]_{\mathfrak{E}}\right]_{0} .
$$

Next we focus on the K-groups of $\mathfrak{E} / \mathfrak{K}_{\mathbb{R}}$. The isomorphism (2) and standard results in K-theory of $\mathrm{C}^{*}$-algebras imply at once that $K_{0}\left(\mathfrak{E} / \mathfrak{K}_{\mathbb{R}}\right)$ and $K_{1}\left(\mathfrak{E} / \mathfrak{K}_{\mathbb{R}}\right)$ are both isomorphic to $\mathbb{Z} \oplus \mathbb{Z}$. More detailed information can be given if we consider the exact sequence

$$
0 \rightarrow S \mathfrak{K}_{\mathbb{Z}} \oplus S \mathfrak{K}_{\mathbb{Z}} \rightarrow C\left(S^{*} \mathbb{S}^{1}, \mathfrak{K}_{\mathbb{Z}}\right) \rightarrow \mathfrak{K}_{\mathbb{Z}} \oplus \mathfrak{K}_{\mathbb{Z}} \rightarrow 0
$$

( $S$ denotes suspension) induced by the $\mathrm{C}^{*}$-algebra homomorphism

$$
\begin{aligned}
\nu: C\left(S^{*} \mathbb{S}^{1}, \mathfrak{K}_{\mathbb{Z}}\right) & \longrightarrow \mathfrak{K}_{\mathbb{Z}} \oplus \mathfrak{K}_{\mathbb{Z}} \\
(f, g) & \longmapsto(f(1), g(1)) .
\end{aligned}
$$

The fact that (10) splits implies that $\nu$ induces a $K_{0}$-isomorphism, while the inclusion of $S \mathfrak{K}_{\mathbb{Z}} \oplus S \mathfrak{K}_{\mathbb{Z}}=\operatorname{ker} \nu$ into $C\left(S^{*} \mathbb{S}^{1}, \mathfrak{K}_{\mathbb{Z}}\right)$ induces a $K_{1}$-isomorphism. We know that if $E$ is any (we will choose one later) rank-one projection on $\ell^{2}(\mathbb{Z})$, then

$$
K_{0}\left(\mathfrak{K}_{\mathbb{Z}}\right)=\mathbb{Z}[E]_{0}
$$

and, by Bott periodicity [25. 11.1.2], $K_{1}\left(C\left(\mathbb{S}^{1}, \mathfrak{K}_{\mathbb{Z}}\right)\right)=\mathbb{Z}[I+(\mathbf{z}-1) E]_{1}$. Let $\mathrm{u}$ and v denote the unitaries in $\left(C\left(S^{*} \mathbb{S}^{1}, \mathfrak{K}_{\mathbb{Z}}\right)\right)^{+}$defined by

$$
\mathrm{u}=(I+(\mathbf{z}-1) E, I) \text { and } \mathrm{v}=(I, I+(\mathbf{z}-1) E) \text {. }
$$


From $C\left(S^{*} \mathbb{S}^{1}, \mathfrak{K}_{\mathbb{Z}}\right) \cong C\left(\mathbb{S}^{1}, \mathfrak{K}_{\mathbb{Z}}\right) \oplus C\left(\mathbb{S}^{1}, \mathfrak{K}_{\mathbb{Z}}\right)$, it follows that:

$$
K_{1}\left(\mathfrak{E} / \mathfrak{K}_{\mathbb{R}}\right) \cong K_{1}\left(C\left(S^{*} \mathbb{S}^{1}, \mathfrak{K}_{\mathbb{Z}}\right)\right)=\mathbb{Z}[\mathrm{u}]_{1} \oplus \mathbb{Z}[\mathrm{v}]_{1},
$$

with the isomorphism in (13) being induced by (2). It also follows from the above remarks that

$$
K_{0}\left(\mathfrak{E} / \mathfrak{K}_{\mathbb{R}}\right) \cong K_{0}\left(C\left(S^{*} \mathbb{S}^{1}, \mathfrak{K}_{\mathbb{Z}}\right)\right) \cong K_{0}\left(\mathfrak{K}_{\mathbb{Z}}\right) \oplus K_{0}\left(\mathfrak{K}_{\mathbb{Z}}\right),
$$

where the second isomorphism is induced by $\nu$, and the first by (2).

Substituting the isomorphism (2) into (5), we get:

$$
0 \rightarrow C\left(S^{*} \mathbb{S}^{1}, \mathfrak{K}_{\mathbb{Z}}\right) \rightarrow \mathfrak{A} / \mathfrak{K}_{\mathbb{R}} \rightarrow \mathfrak{A} / \mathfrak{E} \rightarrow 0 .
$$

Substituting (8), (91), (13) and (14) into the cyclic exact sequence in K-theory associated to (15) we get:

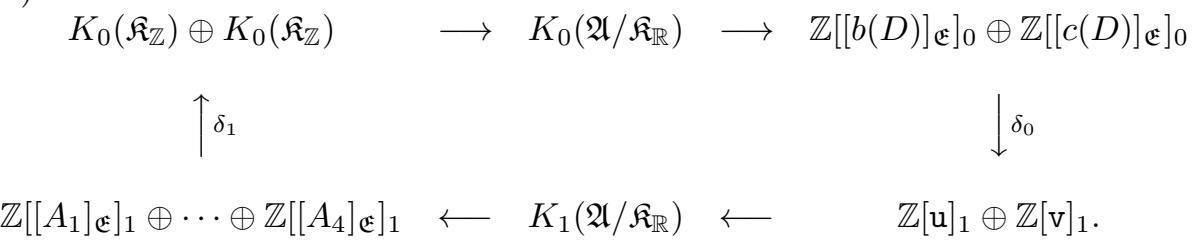

Our notation does not distinguish between $\delta_{0}\left(\right.$ or $\left.\delta_{1}\right)$ in (16) and the exponential mapping $\delta_{0}: K_{0}(\mathfrak{A} / \mathfrak{E}) \rightarrow K_{1}\left(\mathfrak{E} / \mathfrak{K}_{\mathbb{R}}\right)$ (or the index mapping $\delta_{1}: K_{1}(\mathfrak{A} / \mathfrak{E}) \rightarrow$ $K_{0}\left(\mathfrak{E} / \mathfrak{K}_{\mathbb{R}}\right)$, respectively) in the cyclic exact sequence of K-groups associated to (5). They are the same, modulo the isomorphism (13) (or (14)). Still, in the proofs of the following two propositions, we will have to be aware of how those isomorphisms are defined.

Proposition 2. In (16), we have $\delta_{0}\left(\left[[b(D)]_{\mathfrak{E}}\right]_{0}\right)=[\mathrm{u}]_{1}+[\mathrm{v}]_{1}=-\delta_{0}\left(\left[[c(D)]_{\mathfrak{E}}\right]_{0}\right)$.

Proof: Although $[c(D)]_{\mathfrak{K}_{\mathbb{R}}}$ is not a projection in $\mathfrak{A} / \mathfrak{K}_{\mathbb{R}}$, it is a self-adjoint lift for $[c(D)]_{\mathfrak{E}}$, since $c(D)$ is self-adjoint already in $\mathfrak{A}$. We therefore have

$$
\delta_{0}\left([c(D)]_{\mathfrak{E}}\right)=-\left[e^{2 \pi i[c(D)]_{\mathfrak{K}_{\mathbb{R}}}}\right]_{1}=-\left[\left[e^{2 \pi i c(D)}\right]_{\mathfrak{G}_{\mathbb{R}}}\right]_{1} .
$$

In view of the isomorphism (2), in order to write the above element of $K_{1}\left(\mathfrak{E} / \mathfrak{K}_{\mathbb{R}}\right)$ as a linear combination of $[\mathrm{u}]_{1}$ and $[\mathrm{v}]_{1}$ we must look at $\gamma_{T}$, where $T=e^{2 \pi i c(D)}=t(D)$, $t(\xi)=e^{2 \pi i c(\xi)}$. By Theorem 2

$$
\gamma_{t(D)}\left(e^{2 \pi i \varphi},+1\right)=\gamma_{t(D)}\left(e^{2 \pi i \varphi},-1\right)=Y_{\varphi} M_{\varphi}^{t} Y_{-\varphi}, \text { for all } \varphi \in \mathbb{R} .
$$

We have chosen $c$ so that

$$
t(\xi)=1 \text { if }|\xi| \geq 1 / 5 .
$$

This implies that

$$
t(j-\varphi)=1 \text { if } 0 \neq j \in \mathbb{Z} \text { and }|\varphi| \leq \frac{1}{2} .
$$

We now choose $E$ as the orthogonal projection onto the subspace of $\ell^{2}(\mathbb{Z})$ generated by $\mathbf{d}=\left(d_{n}\right)_{n \in \mathbb{Z}}, d_{n}=0$ if $n \neq 0$, and $d_{0}=1$. It follows from (19) that

$$
M_{\varphi}^{t}=I+[t(-\varphi)-1] E \text { if }|\varphi| \leq \frac{1}{2} .
$$


Let us choose a smooth $h: \mathbb{R} \rightarrow \mathbb{R}$ which vaninshes on $[-1 / 4,+1 / 4]$. For each $x \in[0,1]$ and each $\varphi \in[-1 / 2,+1 / 2]$, define

$$
U_{x}(\varphi)=Y_{(1-x) \varphi+x h(\varphi)}[I+(t(-\varphi)-1) E] Y_{(x-1) \varphi-x h(\varphi)} .
$$

$x \mapsto U_{x}$ is homotopy of unitaries in $C\left([-1 / 2,+1 / 2], \mathfrak{K}_{\mathbb{Z}}\right)^{+}$satisfying

$$
U_{0}(\varphi)=Y_{\varphi} M_{\varphi}^{t} Y_{-\varphi} \text { and } U_{1}(\varphi)=Y_{h(\varphi)}[I+(t(-\varphi)-1) E] Y_{-h(\varphi)},
$$

for all $\varphi \in[-1 / 2,+1 / 2]$. Since $Y_{h(\varphi)}=I$ if $|\varphi| \leq 1 / 4$ and $t(-\varphi)-1=0$ if $|\varphi| \geq 1 / 5$, we have $U_{1}(\varphi)=I+(t(-\varphi)-1) E$ for all $\varphi \in[-1 / 2,+1 / 2]$. Now $U_{1}(\varphi)=I$ for $|\varphi| \geq 1 / 5$ implies also that

$$
V_{1}(z)=I+[g(z)-1] E, \quad z \in \mathbb{S}^{1},
$$

with $g\left(e^{2 \pi i \varphi}\right)=t(-\varphi)$ if $\varphi \in[-1 / 2,+1 / 2]$, defines a unitary $V_{1} \in C\left(\mathbb{S}^{1}, \mathfrak{K}_{\mathbb{Z}}\right)^{+}$ homotopic to $z \mapsto \gamma_{t(D)}(z,+1)=\gamma_{t(D)}(z,-1)$ (because of (17)). Since the winding number of $g$ around the origin is $+1, V_{1}$ is homotopic to $I+(\mathbf{z}-1) E$. This shows that

$$
\delta_{0}\left(\left[[c(D)]_{\mathfrak{E}}\right]_{0}\right)=-[(I+(\mathbf{z}-1) E, I+(\mathbf{z}-1) E)]_{1}=-\left([\mathrm{u}]_{1}+[\mathrm{v}]_{1}\right) .
$$

Analogously, one can show that

$$
\delta_{0}\left(\left[[b(D)]_{\mathfrak{E}}\right]_{0}\right)=-[(I+(\overline{\mathbf{z}}-1) E, I+(\overline{\mathbf{z}}-1) E)]_{1}=[\mathrm{u}]_{1}+[\mathrm{v}]_{1},
$$

as we wanted.

Up to here we have understood $K_{0}$-elements as formal differences of classes of self-adjoint idempotents and $K_{1}$-elements as classes of unitaries. At this point, however, it is more convenient to use Banach-algebra K-theory definitions: $K_{0}$ will now consist of formal differences of classes of idempotents, and $K_{1}$ of classes of invertible elements. These two descriptions of the K-groups are equivalent for any $\mathrm{C}^{*}$-algebra [2]. Allowing $K_{0}$-elements to be represented by non-selfadjoint idempotents yields the very explicit expression for the index mapping given in the following lemma, which has been used before in 21, and is used below in our computation (Proposition 3) of the index mapping $\delta_{1}$ in (16).

Lemma 1. Let $A$ be a unital Banach algebra and let $\delta_{1}: K_{1}(A / J) \rightarrow K_{0}(J)$ denote the index mapping in the standard $K$-theory cyclic exact sequence associated to the short exact sequence of Banach algebras $0 \rightarrow J \rightarrow A \stackrel{\pi}{\rightarrow} A / J \rightarrow 0$. If $u \in M_{n}(A / J)$ is an invertible, $\pi(a)=u$ and $\pi(b)=u^{-1}$, then

$$
\delta_{1}\left([u]_{1}\right)=\left[\left(\begin{array}{cc}
2 a b-(a b)^{2} & a(2-b a)(1-b a) \\
(1-b a) b & (1-b a)^{2}
\end{array}\right)\right]_{0}-\left[\left(\begin{array}{ll}
1 & 0 \\
0 & 0
\end{array}\right)\right]_{0} .
$$

Proof: Let $w \in M_{2 n}(A)$ be defined by

$$
w=\left(\begin{array}{cc}
2 a-a b a & a b-1 \\
1-b a & b
\end{array}\right) .
$$

Then $w$ is invertible,

$$
w^{-1}=\left(\begin{array}{cc}
b & 1-b a \\
a b-1 & 2 a-a b a
\end{array}\right) \text { and } \pi(w)=\left(\begin{array}{cc}
u & 0 \\
0 & u^{-1}
\end{array}\right) .
$$

Our claim now follows immediately from [2, 8.3.1]. 
Proposition 3. Let $A \in \mathfrak{A}$ be such that $[A]_{\mathfrak{E}}$ is invertible in $\mathfrak{A} / \mathfrak{E}$. Then $\gamma_{A}(z,-1)$ and $\gamma_{A}(z,+1)$ are Fredholm operators in $\mathfrak{B}$ for every $z \in \mathbb{S}^{1}$ and

$$
\delta_{1}\left(\left[[A]_{\mathfrak{E}}\right]_{1}\right)=\left(\operatorname{ind}\left(\gamma_{A}(1,-1)\right)[E]_{0} \text {, ind }\left(\gamma_{A}(1,+1)\right)[E]_{0}\right) \in K_{0}\left(\mathfrak{K}_{\mathbb{Z}}\right) \oplus K_{0}\left(\mathfrak{K}_{\mathbb{Z}}\right)
$$

where ind denotes the Fredholm index, and $E$ is as in (12).

Proof: Let $B \in \mathfrak{A}$ be such that $I-A B$ and $I-B A$ belong to $\mathfrak{E}$. By Theorem 3 $\gamma_{I-A B}=\gamma_{I}-\gamma_{A} \gamma_{B}$ and $\gamma_{I-B A}=\gamma_{I}-\gamma_{B} \gamma_{A}$ belong to $C\left(S^{*} \mathbb{S}^{1}, \mathfrak{K}_{\mathbb{Z}}\right)$; i.e., for each $z \in \mathbb{S}^{1}, \gamma_{B}(z, \pm 1)$ is an inverse modulo $\mathfrak{K}_{\mathbb{Z}}$ for $\gamma_{A}(z, \pm 1)$, what proves the first assertion.

Let us take $a=[A]_{\mathfrak{K}_{\mathbb{R}}}$ as a lift (pre-image) for $u=[A]_{\mathfrak{E}}$ with respect to the canonical mapping $\mathfrak{A} / \mathfrak{K}_{\mathbb{R}} \rightarrow \mathfrak{A} / \mathfrak{E}$. Let $B \in \mathfrak{A}$ be such that $[B]_{\mathfrak{E}}=u^{-1}$. Then $b=[B]_{\mathfrak{K}_{\mathbb{R}}}$ is a lift for $u^{-1}$. Applying Lemma 1 and using the isomorphism (14), we see that $\delta_{1}\left([\mathrm{u}]_{1}\right)$ is equal to

$$
\begin{aligned}
& \left(\left[\begin{array}{cc}
2 \mathrm{~A}^{-} \mathrm{B}^{-}-\left(\mathrm{A}^{-} \mathrm{B}^{-}\right)^{2} & \mathrm{~A}^{-}\left(2 I-\mathrm{B}^{-} \mathrm{A}^{-}\right)\left(I-\mathrm{B}^{-} \mathrm{A}^{-}\right) \\
\left(I-\mathrm{B}^{-} \mathrm{A}^{-}\right) \mathrm{B}^{-} & \left(I-\mathrm{B}^{-} \mathrm{A}^{-}\right)^{2}
\end{array}\right)\right]_{0}-\left[\left(\begin{array}{cc}
I & 0 \\
0 & 0
\end{array}\right)\right]_{0}, \\
& \left.\left[\left(\begin{array}{cc}
2 \mathrm{~A}^{+} \mathrm{B}^{+}-\left(\mathrm{A}^{+} \mathrm{B}^{+}\right)^{2} & \mathrm{~A}^{+}\left(2 I-\mathrm{B}^{+} \mathrm{A}^{+}\right)\left(I-\mathrm{B}^{+} \mathrm{A}^{+}\right) \\
\left(I-\mathrm{B}^{+} \mathrm{A}^{+}\right) \mathrm{B}^{+} & \left(I-\mathrm{B}^{+} \mathrm{A}^{+}\right)^{2}
\end{array}\right)\right]_{0}-\left[\left(\begin{array}{cc}
I & 0 \\
0 & 0
\end{array}\right)\right]_{0}\right),
\end{aligned}
$$

where $\mathrm{A}^{ \pm}=\gamma_{A}(1, \pm 1)$ and $\mathrm{B}^{ \pm}=\gamma_{B}(1, \pm 1)$.

Let us recall that the index mapping $\delta_{F}$ for the exact sequence

$$
0 \longrightarrow \mathfrak{K}_{\mathbb{Z}} \longrightarrow \mathfrak{B} \longrightarrow \mathfrak{B} / \mathfrak{K}_{\mathbb{Z}} \longrightarrow 0
$$

"is" the Fredholm index [25, 9.4.2], in the sense that $\delta_{F}$ maps $\left[[T]_{\mathfrak{K}_{\mathbb{Z}}}\right]_{1}$, if $T \in \mathfrak{B}$ is a Fredholm operator, into ind $(T)[E]_{0}$. On the other hand, again by Lemma 1 the first component in the expression for $\delta_{1}\left([\mathrm{u}]_{1}\right)$ in (21) equals $\delta_{F}\left(\left[\left[\mathrm{~A}^{-}\right]_{\mathfrak{K}_{\mathbb{Z}}}\right]_{1}\right)$. I.e., that first component is equal to ind $\left(\mathrm{A}^{-}\right)[E]_{0}$, as we wanted. Analogously for the other component.

Corollary 2. In (16), we have $\delta_{1}\left(\left[\left[A_{1}\right]_{\mathfrak{E}}\right]_{1}\right)=\left(0,[E]_{0}\right), \delta_{1}\left(\left[\left[A_{2}\right]_{\mathfrak{E}}\right]_{1}\right)=\left([E]_{0}, 0\right)$, $\delta_{1}\left(\left[\left[A_{3}\right]_{\mathfrak{E}}\right]_{1}\right)=\left(0,-[E]_{0}\right)$ and $\delta_{1}\left(\left[\left[A_{4}\right]_{\mathfrak{E}}\right]_{1}\right)=\left(-[E]_{0}, 0\right)$.

Proof: It takes a straighforward application of all definitions to write down explicit formulas for $\gamma_{A_{i}}(1, \pm 1), i=1, \cdots, 4$. Their indices can be computed by [10. 19.1.14], for example. We omit the details, which can be found in [26].

We are now ready to conclude the analysis of (16). It follows from Corollary 2 that $\delta_{1}$ is surjective, and hence the upper right arrow in (16), which maps $\left[[I]_{\mathfrak{K}_{\mathbb{R}}}\right]_{0}$ to $\left[[I]_{\mathfrak{E}}\right]_{0}$, is injective. By Corollary $1[b(D)]_{\mathfrak{E}}$ and $[c(D)]_{\mathfrak{E}}$ are mutually orthogonal projections in $\mathfrak{A} / \mathfrak{E}$, hence:

$$
\left[[b(D)]_{\mathfrak{E}}\right]_{0}+\left[[c(D)]_{\mathfrak{E}}\right]_{0}=\left[[b(D)+c(D)]_{\mathfrak{E}}\right]_{0}=\left[[I]_{\mathfrak{E}}\right]_{0} .
$$

By Proposition 2 the kernel of $\delta_{0}$ is generated by $\left[[b(D)]_{\mathfrak{E}}\right]_{0}+\left[[c(D)]_{\mathfrak{E}}\right]_{0}$, and this shows that

$$
K_{0}\left(\mathfrak{A} / \mathfrak{K}_{\mathbb{R}}\right)=\mathbb{Z}\left[[I]_{\mathfrak{K}_{\mathbb{R}}}\right]_{0} .
$$

The image of $\delta_{0}$ is complemented by $\mathbb{Z}[\mathrm{u}]_{1}$ (by Proposition 2) and the kernel of $\delta_{1}$ is equal to $\mathbb{Z}\left(\left[\left[A_{1}\right]_{\mathfrak{E}}\right]_{1}+\left[\left[A_{3}\right]_{\mathfrak{E}}\right]_{1}\right) \oplus \mathbb{Z}\left(\left[\left[A_{2}\right]_{\mathfrak{E}}\right]_{1}+\left[\left[A_{4}\right]_{\mathfrak{E}}\right]_{1}\right)$ (by Corollary 2). The exactness of (16) then implies that

$$
K_{1}\left(\mathfrak{A} / \mathfrak{K}_{\mathbb{R}}\right)=\mathbb{Z}\left[\left[B_{1}\right]_{\mathfrak{K}_{\mathbb{R}}}\right]_{1} \oplus \mathbb{Z}\left[\left[B_{2}\right]_{\mathfrak{K}_{\mathbb{R}}}\right]_{1} \oplus \mathbb{Z}\left[\left[B_{3}\right]_{\mathfrak{K}_{\mathbb{R}}}\right]_{1},
$$

if $B_{1} \in \mathfrak{A}, B_{2} \in \mathfrak{A}$ and $B_{3} \in \mathfrak{E}^{+}$are such that

$$
\sigma_{B_{1}}=\sigma_{A_{1}} \cdot \sigma_{A_{3}}=(l, l), \quad \sigma_{B_{2}}=\sigma_{A_{2}} \cdot \sigma_{A_{4}}=(\tilde{l}, \tilde{l})
$$


and

$$
\left[\gamma_{B_{3}}\right]_{1}=[\mathrm{u}]_{1}
$$

If we take $B_{1}=l(M)$ and $B_{2}=\tilde{l}(M)$, then we obviously get (22). We claim that

$$
B_{3}=b(M) t(D)+c(M),
$$

$t$ as in the proof of Proposition 2 satisfies (23). That follows from

$$
\gamma_{B_{3}}\left(e^{2 \pi i \varphi},+1\right)=Y_{\varphi} M_{\varphi}^{t} Y_{-\varphi}, \quad \gamma_{B_{3}}\left(e^{2 \pi i \varphi},-1\right)=I
$$

and the fact, shown in the proof of Proposition 2 that the unitary

$$
e^{2 \pi i \varphi} \mapsto Y_{\varphi} M_{\varphi}^{t} Y_{-\varphi}
$$

is homotopic, within $C\left(\mathbb{S}^{1}, \mathfrak{K}_{\mathbb{Z}}\right)^{+}$, to $I+(\mathbf{z}-1) E$. This settles the question of computing the K-theory of $\mathfrak{A} / \mathfrak{K}_{\mathbb{R}}$.

The Fredholm index of $B_{3}$ is $(-1)$, by Theorem [5] The index mapping in the six-term exact sequence associated to $0 \rightarrow \mathfrak{K}_{\mathbb{R}} \rightarrow \mathfrak{A} \rightarrow \mathfrak{A} / \mathfrak{K}_{\mathbb{R}} \rightarrow 0$ is therefore surjective. This implies that the canonical projection $\mathfrak{A} \rightarrow \mathfrak{A} / \mathfrak{K}_{\mathbb{R}}$ induces isomorphisms between $K_{0}(\mathfrak{A})$ and $K_{0}\left(\mathfrak{A} / \mathfrak{K}_{\mathbb{R}}\right)$ and between $K_{1}(\mathfrak{A})$ and the kernel of the index mapping $K_{1}\left(\mathfrak{A} / \mathfrak{K}_{\mathbb{R}}\right) \rightarrow \mathbb{Z}$, which is the subgroup generated by $\left[\left[B_{1}\right]_{\mathfrak{K}_{\mathbb{R}}}\right]_{1}$ and $\left[\left[B_{2}\right]_{\mathfrak{K}_{\mathbb{R}}}\right]_{1}$ (since $B_{1}$ and $B_{2}$ are invertible). This finishes the proof of:

Theorem 6. $K_{0}(\mathfrak{A})=\mathbb{Z}[I]_{0}$ and $K_{1}(\mathfrak{A})=\mathbb{Z}[l(M)]_{1} \oplus \mathbb{Z}[\tilde{l}(M)]_{1}$.

\section{The OPERATOR-VALUED SYMBOL EXACT SEQUENCE}

Let $\mathfrak{A}^{\diamond}$ denote the $\mathrm{C}^{*}$-algebra of bounded operators on $L^{2}(\mathbb{R})$ generated by all operators of type (2) or (3) (listed at the beginning of this paper). In this section, we define a surjective $\mathrm{C}^{*}$-algebra homomorphism

$$
\psi: \mathfrak{A} \longrightarrow \mathfrak{A}^{\diamond} \oplus \mathfrak{A}^{\diamond}
$$

and exhibit a $\mathrm{C}^{*}$-algebra isomorphism between the images of $\gamma$ and $\psi$

$$
\iota: \operatorname{Im} \gamma \longrightarrow \operatorname{Im} \psi
$$

such that $\psi=\iota \circ \gamma$. We compute the K-theory of $\mathfrak{A}^{\diamond}$, and then analyse the standard cyclic exact sequence associated to $\psi$ and $\gamma$.

Given $u \in L^{2}(\mathbb{R})$, for almost every $\varphi \in \mathbb{R}$, the sequence $u^{\diamond}(\varphi)=(u(\varphi-j))_{j \in \mathbb{Z}}$ belongs to $\ell^{2}(\mathbb{Z})$. A unitary mapping

$$
W: L^{2}(\mathbb{R}) \longrightarrow L^{2}\left(\mathbb{S}^{1} ; \ell^{2}(\mathbb{Z})\right)
$$

is defined by $(W u)\left(e^{2 \pi i \varphi}\right)=Y_{\varphi} u^{\diamond}(\varphi)$. Wu is smooth whenever $u$ is. Given a continuous function $g \in C\left(\mathbb{S}^{1}, \mathfrak{B}\right)$, let $\mu(g)$ denote the bounded operator on $L^{2}\left(\mathbb{S}^{1} ; \ell^{2}(\mathbb{Z})\right)$ of multiplication by $g$. An injective $\mathrm{C}^{*}$-algebra homomorphism is defined by

$$
\begin{array}{cccc}
\mu \oplus \mu: C\left(S^{*} \mathbb{S}^{1}, \mathfrak{B}\right) & \longrightarrow & \mathcal{L}\left(L^{2}\left(\mathbb{S}^{1} ; \ell^{2}(\mathbb{Z})\right)\right) \oplus \mathcal{L}\left(L^{2}\left(\mathbb{S}^{1} ; \ell^{2}(\mathbb{Z})\right)\right) \\
(f, g) & \longmapsto & (\mu(f), \mu(g))
\end{array}
$$

(we have used notation explained just before Proposition 11). It is straightforward to check that, given $A \in \mathfrak{A}^{\diamond}$ and $d \in C[-\infty,+\infty]$, we have

$$
\mu \oplus \mu\left(\gamma_{B}\right)=\left(d(-\infty) W F^{-1} A F W^{-1}, d(+\infty) W F^{-1} A F W^{-1}\right),
$$

if $B=d(M) A$. The set of all such $B$ 's and the compact ideal together generate a dense subalgebra of $\mathfrak{A}$. Since the compacts are contained in the kernel of $\gamma$, we get: 
Theorem 7. There exists a surjective $C^{*}$-algebra homomorphism

$$
\psi: \mathfrak{A} \longrightarrow \mathfrak{A}^{\diamond} \oplus \mathfrak{A}^{\diamond}
$$

such that $\psi(A)=(A, A)$ if $A \in \mathfrak{A}^{\diamond}, \psi(a(M))=(a(-\infty) I, a(+\infty) I)$ if $a \in$ $C([-\infty,+\infty])$ and $\psi(K)=0$ if $K$ is compact. Denoting by $\iota$ the conjugation by $F W^{-1} \oplus F W^{-1}$ composed with $\mu \oplus \mu$, we have $\iota \circ \gamma=\psi$.

Thus $\operatorname{Im} \gamma$ is isomorphic to $\mathfrak{A}^{\diamond} \oplus \mathfrak{A}^{\diamond}$. We now compute the K-theory of $\mathfrak{A}^{\diamond}$.

Theorem 8. $K_{0}\left(\mathfrak{A}^{\diamond}\right)=\mathbb{Z}[I]_{0}$ and $K_{1}\left(\mathfrak{A}^{\diamond}\right)=\mathbb{Z}\left[e^{i M}\right]_{1}$, where $e^{i M}$ denotes the operator of multiplication by $x \mapsto e^{i x}$.

Proof: Let $\alpha$ denote the automorphism of $A=C[-\infty,+\infty]$ defined by

$$
[\alpha(f)](x)=f(x-1), x \in \mathbb{R}, f \in A,
$$

and let $A \times{ }_{\alpha} \mathbb{Z}$ denote the envelopping $\mathrm{C}^{*}$-algebra $[8$ of the Banach algebra with involution $\ell^{1}(\mathbb{Z}, A)$ of all summable $\mathbb{Z}$-sequences in $A$ equipped with the product

$$
(\mathbf{f} \cdot \mathbf{g})(n)=\sum_{k \in \mathbb{Z}} f_{k} \alpha^{k}\left(g_{n-k}\right), \quad n \in \mathbb{Z}, \mathbf{f}=\left(f_{k}\right)_{k \in \mathbb{Z}}, \mathbf{g}=\left(g_{k}\right)_{k \in \mathbb{Z}},
$$

and involution $\mathbf{f}^{*}(n)=\alpha^{n}\left(\bar{f}_{-n}\right)$.

The K-groups of $A$ and $A \times_{\alpha} \mathbb{Z}$ fit 22 into the Pimsner-Voiculescu exact sequence

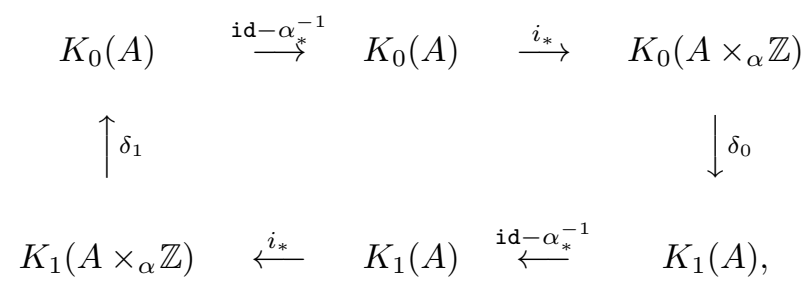

where id denotes the identity on K-groups and $i$ the canonical inclusion of $A$ into $A \times_{\alpha} \mathbb{Z}$ : $i(a)$ is the sequence whose only nonvanishing entry is $a$ at position zero. Abusing notation, we will denote $i(a)$ by $a$. Using that $K_{0}(A)=\mathbb{Z}[1]_{0}$, that $K_{1}(A)=0$, and that $\alpha(1)=1$, it follows easily from (24) that $K_{0}\left(A \times{ }_{\alpha} \mathbb{Z}\right)=\mathbb{Z}[1]_{0}$ and that $K_{1}\left(A \times_{\alpha} \mathbb{Z}\right)$ is also isomorphic to $\mathbb{Z}$.

For each integer $k$, let $\mathbf{d}_{k}$ denote the sequence whose only nonvanishing entry is 1 at position $k . \mathbf{d}_{1}$ is a unitary in $A \times{ }_{\alpha} \mathbb{Z}$ such that, for every $a \in A, \alpha(a)=$ $\mathbf{d}_{1} a \mathbf{d}_{1}^{*}$. It then follows from the equation at the beginning of [22, page 102] that $K_{1}\left(A \times_{\alpha} \mathbb{Z}\right)=\mathbb{Z}\left[\mathbf{d}_{-1}\right]_{1}$.

To prove the theorem, it therefore suffices to show that there exists a $\mathrm{C}^{*}$-algebra isomorphism $\varphi: A \times_{\alpha} \mathbb{Z} \rightarrow \mathfrak{A}^{\diamond}$ such that $\varphi\left(\mathbf{d}_{-1}\right)=e^{i M}$.

For sequences $\mathbf{a}=\left(a_{j}\right)_{j \in \mathbb{Z}}$ with only a finite number of nonzero entries, define

$$
\varphi(\mathbf{a})=\sum_{j} a_{j}(D) e^{-i j M}
$$

It is elementary to check that $\varphi$ extends to a ${ }^{*}$-homomorphism from $\ell^{1}(\mathbb{Z}, A)$ to $\mathfrak{A}^{\diamond}$, which obviously satisfies $\varphi\left(\mathbf{d}_{-1}\right)=e^{i M}$. It then follows from [8, 2.7.4] that $\varphi$ extends to a $\mathrm{C}^{*}$-algebra homomorphism $\varphi: A \times{ }_{\alpha} \mathbb{Z} \rightarrow \mathfrak{A}^{\diamond}$. The image of $\varphi$ is dense, by definition of $\mathfrak{A}^{\diamond}$. So, all that remains to be shown is that $\varphi$ is injective.

Given $z \in \mathbb{S}^{1}$ and $\mathbf{a}=\left(a_{j}\right)_{j \in \mathbb{Z}} \in \ell^{1}(\mathbb{Z}, A)$, define $\gamma_{z}(\mathbf{a})=\left(z^{j} a_{j}\right)_{j \in \mathbb{Z}} \cdot \gamma_{z}$ extends to an automorphism of $A \times_{\alpha} \mathbb{Z}$ and $z \mapsto \gamma_{z}$ is an action of the circle. For each 
$z \in \mathbb{S}^{1}$, let $U_{z}$ denote the unitary mapping on $L^{2}(\mathbb{R})$ given by $\left(U_{z} f\right)(x)=z^{x} f(x)$, $x \in \mathbb{R}$, and define $\beta_{z}(A)=F U_{z} F^{-1} A F U_{z}^{-1} F^{-1}, A \in \mathfrak{A}^{\diamond} . z \mapsto \beta_{z}$ is an action of $\mathbb{S}^{1}$ by automorphisms on $\mathfrak{A}^{\diamond}$ satisfying $\beta_{z} \circ \varphi=\varphi \circ \gamma_{z}$ for each $z$. To show that $\varphi$ is injective, it therefore suffices to show that its restriction to the fixed points of $\gamma$ is injective (by [9, Proposition 2.9]).

Let $C$ denote the algebra of points fixed by $\gamma$,

$$
C=\left\{\mathbf{x} \in A \times{ }_{\alpha} \mathbb{Z} ; \gamma_{z}(\mathbf{x})=\mathbf{x} \text { for all } z \in \mathbb{S}^{1}\right\},
$$

and let $E: A \times_{\alpha} \mathbb{Z} \rightarrow A \times_{\alpha} \mathbb{Z}$ be defined by

$$
E(\mathbf{x})=\int_{\mathbb{S}^{1}} \gamma_{z}(\mathbf{x}) d z, \not\left(e^{i \theta}\right)=d \theta / 2 \pi .
$$

If $\mathbf{x} \in C$, then $E(\mathbf{x})=\mathbf{x}$; hence $C$ is contained in the image of $E, \operatorname{Im} E$. Since, for all $j \neq 0$ the integral $\int z^{j} d z$ vanishes, we get $E(\mathbf{a}) \in A$ for all $\mathbf{a} \in \ell^{1}(\mathbb{Z}, A)$. Since $A$ is a closed subalgebra of $A \times_{\alpha} \mathbb{Z}$ and $\ell^{1}(\mathbb{Z}, A)$ is dense in $A \times_{\alpha} \mathbb{Z}$, we get $\operatorname{Im} E \subseteq A$. Since $A \subseteq C$, we get $A=C$. But it is clear that $\varphi$ restricted to $A$ is injective, as we wanted.

Let $\mathfrak{J}$ denote the $\mathrm{C}^{*}$-algebra of bounded operators on $L^{2}(\mathbb{R})$ generated by all operators of the form $a(M) b(D)$, for $a \in C_{0}(\mathbb{R})$ and $b \in C[-\infty,+\infty]$. Choose a sequence $\chi_{j} \in C_{0}(\mathbb{R})$ such that $0 \leq \chi_{j} \leq 1$, the support of each $\chi_{j}$ is contained in the interval $(-j,+j)$ and $\chi_{j}(x) \rightarrow 1$ for all $x \in \mathbb{R}$. Using the facts stated in the Introduction about the commutators $[a(M), b(D)]$, it is straightforward to prove (this is a particular case of [4, Lemma VII.1.2]) that a given $A \in \mathfrak{A}$ belongs to $\mathfrak{J}$ if and only if there exists a sequence of compact operators $C_{j}$ such that $\chi_{j}(M) A+C_{j} \rightarrow A$. This implies that $\gamma$ vanishes on a dense subset of $\mathfrak{J}$; and hence $\mathfrak{J} \subset \operatorname{ker} \gamma$. It also shows that $A \in \mathfrak{J}$ if and only if $\sigma_{A}$ vanishes over all points of the form $(( \pm \infty, z), \pm \infty), z \in \mathbb{S}^{1}$. Since the supremum of $\left|\sigma_{A}\right|$ over all those points is bounded by $\left\|\gamma_{A}\right\|$ [7. Proposition 3.4], we then get

$$
\mathfrak{J}=\operatorname{ker} \gamma .
$$

The restriction of $\sigma$ to $\mathfrak{J}$ induces an isomorphism

$$
\mathfrak{J} / \mathfrak{K}_{\mathbb{R}} \cong C_{0}(\mathbb{R} \times\{-\infty,+\infty\}) .
$$

This follows from a general result [4, Theorem VI.2.2] about comparison algebras ( $\mathfrak{J}$ is the minimal comparison algebra over $\mathbb{R}$ ). A direct proof for our case is given in 26. Lema 1.15].

If we now quotient the exact sequence $0 \rightarrow \operatorname{ker} \gamma \rightarrow \mathfrak{A} \rightarrow \operatorname{Im} \gamma \rightarrow 0$ by the compacts and use Theorem 7 and (25), we obtain the exact sequence of $\mathrm{C}^{*}$-algebras

$$
0 \longrightarrow \mathfrak{J} / \mathfrak{K}_{\mathbb{R}} \stackrel{\iota}{\longrightarrow} \mathfrak{A} / \mathfrak{K}_{\mathbb{R}} \stackrel{\psi}{\longrightarrow} \mathfrak{A}^{\diamond} \oplus \mathfrak{A}^{\diamond} \longrightarrow 0 .
$$

and its associated six-term exact sequence

$$
\begin{array}{cccccc}
0 & \longrightarrow & K_{0}\left(\mathfrak{A} / \mathfrak{K}_{\mathbb{R}}\right) & \stackrel{\psi_{*}}{\longrightarrow} & K_{0}\left(\mathfrak{A}^{\diamond} \oplus \mathfrak{A}^{\diamond}\right) \\
\uparrow & & & & \\
K_{1}\left(\mathfrak{A}^{\diamond} \oplus \mathfrak{A}^{\diamond}\right) & \stackrel{\psi_{*}}{\longleftarrow} & K_{1}\left(\mathfrak{A} / \mathfrak{K}_{\mathbb{R}}\right) & \stackrel{\iota_{*}}{\longleftarrow} & K_{1}\left(\mathfrak{J} / \mathfrak{K}_{\mathbb{R}}\right) .
\end{array}
$$

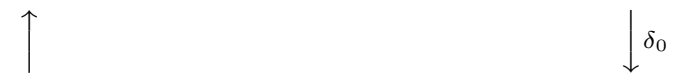


It follows from Theorem 8 that $K_{0}\left(\mathfrak{A}^{\diamond} \oplus \mathfrak{A}^{\diamond}\right)=\mathbb{Z}[(I, 0)]_{0} \oplus \mathbb{Z}[(0, I)]_{0}$. Since $\psi(I)=(I, I)$, we have

$$
[(I, 0)]_{0}+[(0, I)]_{0} \in \operatorname{ker} \delta_{0} .
$$

The isomorphism (26) implies that $\left[\left[U_{+}\right]_{\mathfrak{K}_{\mathbb{R}}}\right]_{1}$ and $\left[\left[U_{-}\right]_{\mathfrak{K}_{\mathbb{R}}}\right]_{1}$ generate $K_{1}\left(\mathfrak{J} / \mathfrak{K}_{\mathbb{R}}\right) \cong$ $\mathbb{Z} \oplus \mathbb{Z}$, if each $U_{ \pm}$is a Fredholm operator in $\mathfrak{J} \oplus \mathbb{C} I$ such that its principal symbol is equal to one on $\mathbb{R} \times\{\mp \infty\}$ and winds once around the origin over $\mathbb{R} \times\{ \pm \infty\}$. We may take, for example, $U_{+}=e^{2 \pi i b(M)} b(D)+c(D)$ and $U_{-}=e^{2 \pi i c(M)} c(D)+b(D)$ ( $b$ and $c$ were defined at the beginning of Section 11). Since the winding numbers of $\bar{\sigma}\left(U_{+}\right)$and $\bar{\sigma}\left(U_{+}\right)$are equal, it follows from Theorem 5 that

$$
i_{*}\left(\left[\left[U_{+}\right]_{\mathfrak{K}_{\mathbb{R}}}\right]_{1}\right)=i_{*}\left(\left[\left[U_{-}\right]_{\mathfrak{K}_{\mathbb{R}}}\right]_{1}\right),
$$

where $i: \mathfrak{J} / \mathfrak{K}_{\mathbb{R}} \rightarrow \mathfrak{C} / \mathfrak{K}_{\mathbb{R}}$ denotes the canonical inclusion. From (30), we then get

$$
\iota_{*}\left(\left[\left[U_{+}\right]_{\mathfrak{K}_{\mathbb{R}}}\right]_{1}\right)=\iota_{*}\left(\left[\left[U_{-}\right]_{\mathfrak{K}_{\mathbb{R}}}\right]_{1}\right)
$$

$\left(\iota: \mathfrak{J} / \mathfrak{K}_{\mathbb{R}} \rightarrow \mathfrak{A} / \mathfrak{K}_{\mathbb{R}}\right.$ denotes the inclusion, as defined in (27)).

The exactness of (28), together with (29) and (31), yields

$$
K_{0}\left(\mathfrak{A} / \mathfrak{K}_{\mathbb{R}}\right)=\mathbb{Z}\left[[I]_{\mathfrak{K}_{\mathbb{R}}}\right]_{0}
$$

and the short exact sequence

$$
0 \longrightarrow \mathbb{Z}\left[\left[U_{+}\right]_{\mathfrak{K}_{\mathbb{R}}}\right]_{1} \stackrel{\iota_{*}}{\longrightarrow} K_{1}\left(\mathfrak{A} / \mathfrak{K}_{\mathbb{R}}\right) \stackrel{\psi_{*}}{\longrightarrow} K_{1}\left(\mathfrak{A}^{\diamond} \oplus \mathfrak{A}^{\diamond}\right) \longrightarrow 0 .
$$

Since $\psi(l(M))=\left(I, e^{i M}\right)$ and $\psi(\tilde{l}(M))=\left(e^{i M}, I\right)$, Theorem 8 and (32) together imply

$$
K_{1}\left(\mathfrak{A} / \mathfrak{K}_{\mathbb{R}}\right)=\mathbb{Z}\left[\left[U_{+}\right]_{\mathfrak{K}_{\mathbb{R}}}\right]_{1} \oplus \mathbb{Z}\left[[l(M)]_{\mathfrak{K}_{\mathbb{R}}}\right]_{1} \oplus \mathbb{Z}\left[[\tilde{l}(M)]_{\mathfrak{K}_{\mathbb{R}}}\right]_{1} .
$$

Since, by Theorem 5 the Fredholm index of $U_{+}$is $(-1)$, this gives another proof of Theorem [6]

\section{ACKNOWLEDGEMENTS}

We are grateful to Ruy Exel for many helpful conversations. It was his the idea of using that $\varphi$ is a covariant homomorphism in the proof of Theorem 8

Severino Melo was partially supported by CNPq (Processo 306214/2003-2), and Cíntia Silva had a scholarship from CAPES during her graduate studies.

\section{REFERENCES}

[1] M. F. Атіуaн \& I. M. Singer, The index of elliptic operators I, Annals of Math. 87 (1968), 484-530.

[2] B. Blackadar, K-theory for operator algebras, Cambridge University Press, Cambridge, 1998.

[3] H. O. CoRdes, Elliptic pseudodifferential operators - an abstract theory, Lecture Notes in Mathematics 756, Springer, Berlin, 1979.

[4] H. O. CoRdes, Spectral theory of linear differential operators and comparison algebras, London Mathematical Society Lecture Note Series 76, Cambridge University Press, Cambridge, 1987.

[5] H. O. Condes, On the two-fold symbol chain of a $C^{*}$-algebra of singular integral operators on a polycilinder, Revista Mat. Iberoamericana 2 (1986), 215-234.

[6] H. O. Cordes \& E. A. Herman, Gelfand theory of pseudodifferential operators, Amer. J. Math 90 (1968), 681-717.

[7] H. O. Cordes \& S. T. Melo, An algebra of singular integral operators with kernels of bounded oscillation and application to periodic differential operators, J. Differential Equations 75-2 (1988), 216-238.

[8] J. Dixmier, Les C*-algèbres et leurs représentations, Gauthier-Villars, Paris, 1969. 
[9] R. Exel, Circle actions on $C^{*}$-algebras, partial automorphisms and a generalized PimsnerVoiculescu exact sequence, J. Funct. Anal. 122-2 (1994), 361-401.

[10] L.Hörmander, The analysis of linear partial differential operators III, Springer, New York, 1990.

[11] R. LAUter, Holomorphic functional calculus in several variables and $\Psi^{*}$-algebras of totally characteristic operators on manifolds with boundary, Thesis, Mainz, 1996.

[12] R. Lauter, Pseudodifferential analysis on conformally compact spaces, Mem. Amer. Math. Soc. 163-777, 2003.

[13] S. T. Melo, Comparison Algebras with Periodic Symbols, Thesis, Berkeley, 1988.

[14] S. T. Melo, A comparison algebra on a cylinder with semi-periodic multiplications, Pacific J. Math. 146-2 (1990), 281-304.

[15] S. T. Melo, R. Nest \& E. Schrohe. $C^{*}$-structure and K-theory of Boutet de Monvel's algebra, J. Reine Angew. Math. 561 (2003), 145-175.

[16] S. T. Melo, T. Schick \& E. Schrohe, A K-theoretic proof of Boutet de Monvel's index theorem, Arxiv, math.KT/0403059 to appear in J. Reine Angew. Math.

[17] R. B. Melrose, The eta invariant and families of pseudodifferential operators, Math. Res. Lett. 2 (1995), 541-561.

[18] R. B. Melrose \& V. Nistor, K-theory of $C^{*}$-algebras of b-pseudodifferential operators, Geom. Funct. Anal. 8-1 (1998), 88-122.

[19] B. Monthubert \& V. Nistor, A topological index theorem for manifolds with corners, Arxiv, math.KT/0507601

[20] S. Moroinnu, K-Theory of suspended pseudo-differential operators, K-Theory 28 (2003), $167-181$.

[21] V. Nistor, Higher index theorems and the boundary map in cyclic cohomology, Doc. Math. 2 (1997), electronic, 263-295.

[22] M. Pimsner \& D. Voiculescu, Exact sequences for K-groups and Ext-groups of certain cross-product $C^{*}$-algebras, J. Operator Theory 4-1 (1980), 93-118.

[23] S. C. Power, Commutator ideals and pseudodifferential $C^{*}$-algebras, Quart. J. Math. Oxford Ser. (2) 31-124 (1980), 467-489.

[24] V. S. RABinovich, On the algebra generated by the pseudodifferential operators on $R^{n}$, the operators of multiplication by almost periodic functions and the shift operators, Soviet Math. Dokl. 25-2 (1982), 498-502.

[25] M. Rørdam, F. Larsen \& N. Laustsen, An Introduction to K-theory for $\mathrm{C}^{*}$-algebras, Cambridge University Press, Cambridge, 2000.

[26] C. C. Silva, K-teoria de operadores pseudodiferenciais com símbolos semiperiódicos, Tese, São Paulo (Arxiv, math.OA/0505261), 2005.

Instituto de Matemática e Estatística, Universidade de São Paulo Caixa Postal 66281, 05311-970 São Paulo, Brazil.

toscano@ime.usp.br, cintia@ime.usp.br 